\title{
A Novel IncRNA, CTD-2017C7.1, Promotes ESCC Tumorigenesis by Binding to PDIA3 and Activating Oncogenic Gene Expressions
}

\section{Liang Wang}

Shenzhen University https://orcid.org/0000-0002-7467-6332

\section{Peikun Ding}

Shenzhen People's Hospital

Kang Zhang

People's Hospital of Wuzhou

\section{Wenhan Yang}

Shenzhen University

\section{Qianqian Chen}

Shenzhen University

\section{Yi Song}

Shenzhen University

\section{Guogang Cheng}

Shenzhen University

\section{Lin Yang}

Shenzhen People's Hospital

\section{Shaowu Ye}

People's Hospital of Wuzhou

\section{Liyuan Sun}

Shenzhen University

Rihong Zhai ( $\nabla$ rhai@szu.edu.cn )

Shenzhen University Health Science Center https://orcid.org/0000-0003-0631-4134

\section{Primary research}

Keywords: CTD-2017C7.1, functional assays, PDIA3, oncogenes, ESCC

Posted Date: June 23rd, 2021

DOI: https://doi.org/10.21203/rs.3.rs-615588/v1 
License: (c) (i) This work is licensed under a Creative Commons Attribution 4.0 International License. Read Full License 


\section{Abstract}

\section{Background}

IncRNAs are dysregulated in many human cancers, including esophageal squamous cell carcinoma (ESCC), and are associated cancer development and progression. In the current study, we aimed to elucidate the biological roles of IncRNA CTD-2017C7.1 in ESCC.

Method

The biological functions of CTD-2017C7.1 were determined in vitro and in vivo. RNA pull-down, MS, RIP, RNA-seq, and qRT-PCR assays were employed to investigate the mechanisms of CTD-2017C7.1.

Results

CTD-2017C7.1 was up-regulated in ESCC tissues and cells, and associated with poor clinical outcome. Overexpression of CTD-2017C7.1 promoted cell proliferation, invasion and migration. CRISPR/Cas9 knockout of CTD-2017C7.1 resulted in reverse effects. Up-regulation of CTD-2017C7.1 also increased ESCC tumor growth in vivo. Mechanistically, CTD-2017C7.1 bound to PDIA3 and activated the expressions of oncogenic genes.

\section{Conclusion}

Our study revealed that CTD-2017C7.1 was an oncogenic IncRNA that may be a potential therapeutic target of ESCC.

\section{Background}

Esophageal cancer ranks as the 8th most-frequent and 6th most-fatal cancer type worldwide[1, 2]. This disease consists of two major histologic subtypes: esophageal adenocarcinoma (EA), which is more prevalent in Western countries; and esophageal squamous cell carcinoma (ESCC), which accounts for $90 \%$ of all cases of esophageal cancer globally and is highly prevalent in Asia, East Africa and South America[3]. In particular, about half of the world's [2]500,000 new ESCC cases were diagnosed annually in China[4]. Since ESCC is asymptomatic in the early stages, most ESCC patients are diagnosed at an advanced stage[2]. As a consequence, the overall five-year survival rate is low $(\sim 20 \%)[5]$. Thus, a better understanding of the molecular events underlying the pathogenesis of ESCC is essential for improving diagnosis and prognostication of ESCC.

LncRNAs are a subtype of noncoding RNAs with more than 200 nucleotides in length. These functional noncoding RNAs are important regulators of gene expression in cis or in trans by diverse mechanisms[68]. Abnormal expression of IncRNAs has been involved in transcriptional regulations, proliferation, apoptosis, metastasis and migration of cells, in a variety of cancer cells[9-11]. Increasing clinical evidence has also indicated that IncRNAs are dysregulated in many human cancers, including ESCC, and 
are associated cancer development and progression[9, 10, 12]. For instance, abnormal expressions of several IncRNAs, such as ZFAS1, PCAT-1, and MALAT1, were associated with ESCC progression[13-15]. Higher expression of the IncRNA ESCCAL-1 was correlated with worse clinicopathologic features in patients with ESCC[16]. Up-regulated of LOC440173 expression was related to histological grade, tumor invasion depth, lymph node metastasis, and TNM stage[17]. High expression of MALAT1 correlates with shorter disease-free survival and overall survival in ESCC patients who underwent radical surgical resection[2]. Interestingly, Inc-MCEl, an oncogenic IncRNA in ESCC, was found to be able to modify the chemosensitivity of ESCC to cisplatin (DDP) both in vivo and in vitro[18]. These observations indicate that IncRNAs play critical roles in ESCC. However, there are still plenty of ESCC-associated IncRNAs have not been identified and functionally characterized.

In our previous study, we have identified a novel IncRNA CTD-2017C7.1 that was abundant expressed in exosomes derived from patients with ESCC[19]. This finding suggested that CTD-2017C7.1 might be involved in the pathogenesis of ESCC. However, the biological roles and the molecular mechanisms of CTD-2017C7.1 in ESCC are still entirely unknown. In this study, we confirmed that CTD-2017C7.1 was upregulated in both ESCC cell lines and tumor tissues. We then conducted loss- and gain-of-function assays to determine the in vitro and in vivo impacts of CTD-2017C7.1 on ESCC tumorigenesis. Moreover, investigations were carried out to assess through which mechanisms that CTD-2017C7.1 might play its regulatory roles in ESCC cells. This study provides new insights into the biological functions of CTD2017C7.1 as well as its regulatory mechanisms in ESCC.

\section{Materials And Methods}

\section{Patient samples}

Nine paired ESCC tumor tissues and corresponding adjacent normal tissues were collected from patients undergoing surgical resection at the Department of Thoracic Surgery, Shenzhen People's Hospital, China. All patients were histologically confirmed by two pathologists. After surgical resection, all tissues were immediately frozen in liquid nitrogen and then stored at $-80^{\circ} \mathrm{C}$ until use. None of these patients were treated with radiotherapy or chemotherapy prior to surgery.

Establishment of CTD-2017C7.1-overexpressing cell lines

To generate ESCC cells in which CTD-2017C7.1 was stably overexpressed, full-length human CTD2017C7.1 (ENSG00000256705.3) was synthesized by Rochen Biotech (Shanghai, China) and was inserted into the EcoRI and Notl sites of pCDH-CMV-MCS-EF1-copGFP-T2A-Puro vector (pCDH, Supplementary Fig. 1A). Then, pCDH-CTD-2017C7.1 (12 $\mu \mathrm{g})$ and packaging vectors (psPAX2, and pMD2.G) were co-transfected into 293T cells (ATCC) using the PEI reagents (Sigma, 408727). The supernatant containing the lentivirus particles was harvested $72 \mathrm{~h}$ after transfection and concentrated using PEG8000 (Beyotime Biotech, Shanghai, China). TE-1 cells were seeded in 12-well plates with $1 \times 10^{5}$ cells per well. Lentivirus were added after TE- 1 attachment. The infected cells were then subjected to 
selection with $5 \mu \mathrm{g} / \mathrm{ml}$ puromycin. The stable transfected cells were harvested after 2 weeks screening. Cell lines with stably expressed CTD-2017C7.1 were confirmed by quantitative real-time PCR (Supplementary Fig. 1B).

Construction of CTD-2017C7.1 CRISPR/Cas9 knockout plasmids

To establish plasmids with silenced CTD-2017C7.1 expression, two guide RNA (gRNA) sequences which were predicted to have knockout effects on CTD-2017C7.1 (Supplementary Fig. 3A-D, 4A-D) were synthesized by GenePharma Co. (Shanghai, China). Then gRNAs were cloned into the pGE-4 (pU6gRNA1U6gRNA2Cas9puro, Supplementary Fig. 2A), using the Bbsl digestive enzyme and the T4 DNA ligase. CTD-2017C7.1 knock-out sequences were verified by sequencing the genomic Cas9 guide-RNA binding site. The primers, gBlocks, and sequence of CTD-2017C7.1 can be found in the Supplementary materials (Supplementary Table 1, Supplementary Fig. 3-4). To construct the knockout cell lines, TE-1 cells were transfected with $10 \mu \mathrm{g}$ CTD-2017C7.1 CRISPR-Cas9 knockout plasmids using the Lipofectamine $2000 \mathrm{kit}$ (Invitrogen,11668-019). After incubation at $37^{\circ} \mathrm{C}$ for $48 \mathrm{~h}$, the knockout cells were further verified by PCR of genomic DNA showing CTD-2017C7.1 gene disruption (Supplementary Fig. 2B).

Subcellular fraction location

Nuclear/Cytosol Fractionation Kit (Phygene, PH1466) was used to separate cytoplasmic and nuclear fraction of TE-1 cells in accordance with supplier's suggestions. Trizol reagent (Invitrogen, CA, USA) was applied to isolate total RNA, which was reverse transcribed in a final volume of $20 \mu \mathrm{l}$ using random primers under conditions for RT reagent kit (TransGen Biotech, Beijing). QPCR was used to detect CTD2017C7.1 levels in cytoplasm and nuclear fraction, using the SYBRGreen PCR kit (TransGen Biotech, Beijing). The expression levels of CTD-2017C7.1 in nuclear and cytoplasmic fraction were normalized to GAPDH, respectively.

Cell culture and transfection

ESCC cell lines (EC109, KYSE180, KYSE70, and TE-1) were obtained from the cell bank of Shanghai Biological Institute (Shanghai, China). Normal esophageal epithelium cells (NE3) were obtained from Pro. Fu L [20]. ESCC cells were cultured in RPMI 1640 (Gibco) with 10\% FBS and NE3 cells were incubated in a 1:1 mixture of EpiLife and dKSFM (Gibco). All of these cells were maintained at $37^{\circ} \mathrm{C}$ in a humidified atmosphere containing $5 \% \mathrm{CO}_{2}$. Cell transfections were performed using Lipofectamine $2000 \mathrm{kit}$ (Invitrogen, CA, USA) according to the manufacturer's instructions.

The authentication of cell lines

TE-1, EC109, KYSE180, and KYSE70 cells were obtained in January, 2018. All these cell lines were tested and authenticated by STR. Genomic DNAs of TE-1, EC109, KYSE180, and KYSE70 cells were extracted and amplified respectively using GenePrint System (Promega). Amplified products were processed using the ABI3730xI Genetic Analyzer. Data were analyzed using GeneMapper4.0 software and then compared 
with the ATCC, DSMZ or JCRB databases for reference matching. The STR results showed that there were no four alleles on the main nine locus, it is certainly no cross contamination of human cells in these cell lines. TE-1, EC109, KYSE180, and KYSE70 cells were tested on Jan.19, 2018 (Supplementary 1-4).

RNA extraction, quantitative realtime PCR (qRT-PCR)

Total RNA was isolated from tissues or cultured cells using Trizol reagent (Invitrogen, CA, USA). Total RNA $(1 \mu \mathrm{g})$ was reverse transcribed to cDNA using random primers with the SuperScript ${ }^{\text {TM }}$ III RT Reagent kit (Invitrogen, CA, USA) following the manufacturer's protocol. QRT-PCR was performed in the CFX96TM Real-Time System (Bio Rad, Hercules, CA, USA). GAPDH was employed as an endogenous control. Results were normalized to the expression of GAPDH. The relative expression of RNAs was calculated using the $2^{-\Delta \Delta C t}$ method. All experiments were performed in triplicate. Sequences of specific primers are listed in Supplementary Table 1.

Cell proliferation assay

Cell proliferation was determined by the cell counting kit-8 (CCK-8) (TransGen Biotech, FC101-02) according to the manufacturer's instructions. Briefly, cells were cultured in a 96 -well plate at $37^{\circ} \mathrm{C}$ for $24 \mathrm{~h}$. Subsequently, $10 \mu \mathrm{l} \mathrm{CCK}-8$ reagent was added into each well and incubated with cells for $2 \mathrm{~h}$. Finally, cell viability was measured every $24 \mathrm{~h}(0,24,48,72 \mathrm{~h})$ using the microplate reader MK3 (Thermo Fisher Scientific, Shanghai, China) at the absorbance of $450 \mathrm{~nm}$. Five replicates were performed for each treatment group.

Cell invasion and migration assays

For the invasion assay, $5 \times 10^{4}$ cells in serum-free RPMI-1640 medium were placed into the upper chamber (Corning Falcon, USA), and $500 \mu \mathrm{L}$ of RPMI-1640 with $10 \%$ FBS was added to the lower chamber. After $24 \mathrm{~h}$ of incubation, the cells on the upper chamber were removed with cotton swabs, and the cells on the lower membrane surface of the membrane that had migrated or invaded from the upper chamber were fixed with $4 \%$ paraformaldehyde for $15 \mathrm{~min}$ and then stained with $0.1 \%$ crystal violet for $10 \mathrm{~min}$. The number of invaded cells was counted under a microscope. All fields were counted randomly in each well.

Cell migration was evaluated by wound healing assay. In brief, cells in 6-well plates were allowed to reach 80-90\% confluence, and wounds were scratched in the cell monolayers using a sterile micropipette tip. Wound closure was recorded using a microscope. The distance across the wound was measured at baseline and at $24 \mathrm{~h}$, and the wound closure was calculated using the following formula: distance of wound closure $=1-$ length of wound $(24 h) /$ length of wound (0h).

In vivo animal study

BALB/CA nude mice (female, 4-6 weeks old) were purchased from the Experimental Animal Center of Guangdong, China; and were maintained under pathogen-free conditions. The mice were randomly divided into two treatment groups ( $n=5$ for each group): one group was injected with cells $\left(1 \times 10^{6}\right)$ of NC 
control cells; another group was injected with cells $\left(1 \times 10^{6}\right)$ of stably expressing CTD-2017C7.1 (in $0.1 \mathrm{ml}$ PBS). Cells were injected subcutaneously into the right flanks of mice. Tumor size was measured every 3 days using a Vernier caliper. Tumor volumes were calculated using the following formula: $0.5 \times \mathrm{L} \times \mathrm{W} 2(\mathrm{~L}$ refers to long and $\mathrm{W}$ refers to short diameters of the tumor). The tumor weight was measured at the endpoint of the study. At 21 days post-injection, mice were euthanized, and tumor were resected and preserved in formaldehyde for HE or IHC staining. The animal study was approved by the Animal Ethical Committee of Shenzhen University Health Science Center. All experimental procedures were manipulated following the approved protocols.

Immunohistochemical (IHC) analysis

IHC assay was conducted to quantify the expression levels of Ki67 protein in the tumor tissues, using Ki67 antibody (Abcam, ab16667) as the primary antibody and HRP-conjugated antibody as the secondary antibody. The detailed procedures for IHC assay have been described previously[21].

RNA pulldown and MS assays

The RNA pull-down assays were performed using the PierceTM Magnetic RNA-Protein Pull-Down Kit (Thermo Fisher Scientific). Briefly, full-length sense and antisense CTD-2017C7.1 sequences were transcribed into TE-1 cells, using the RNA 3' End Desthiobiotinylation Kit (Thermo Fisher Scientific) and RNA Production System-T7 (Promega, Madison, WI, USA), respectively. Cells were isolated and lysed using a protein lysis buffer. The biotin-labeled probe was captured using the streptavidin magnetic beads. Then the lysates of TE- 1 cells were incubated with biotinylated nucleic acid compounds. The retrieved proteins were purified and detected by silver staining and the eluted RNA-binding proteins complex were subjected to mass spectrometry analysis. For LC-MS, proteins were digested by trypsin and separated by liquid chromatography, then detected by Q-Exactive MS (Thermo Fisher Scientific, Hudson, NH).

RNA immunoprecipitation (RIP)

RNA immunoprecipitation (RIP) analysis was performed using the Imprint ${ }^{\circledR}$ RNA Immunoprecipitation kit (Sigma, RIP-12Rxn) in accordance with the user manual. In brief, TE- 1 cells $\left(1 \times 10^{7}\right)$ were treated with RIP lysis buffer to obtain the cell extract. The cell lysis was centrifuged at $13,000 \mathrm{~g}$ at $4^{\circ} \mathrm{C}$ for $10 \mathrm{~min}$. Then the collected supernatant was incubated with RIP buffer containing magnetic beads conjugated to specific antibody or normal anti-rabbit IgG at $4^{\circ} \mathrm{C}$ overnight. IgG was considered as the negative control. Subsequently, beads were incubated with proteinase K Buffer and RIP wash buffer to remove proteins. RNA was purified by phenol and chloroform. The purified RNAs were furthered subjected to qRT-PCR analysis to assess the expression of precipitated CTD-2017C7.1.

RNA Sequencing (RNA-seq)

To investigate the target genes regulated by CTD-2017c7.1, total RNA was extracted from TE-1 cells stably expressed CTD-2017C7.1 or NC control cells, using Trizol reagent (Invitrogen, CA, USA). RNA 
quality was assessed using the NanoPhotometer ${ }^{\circledR}$ spectrophotometer (IMPLEN, CA, USA). The NEBNext ${ }^{\circledR}$ UltraTM RNA Library Prep Kit for Illumina ${ }^{\circledR}$ (NEB, USA) was used for generating the sequencing libraries. In brief, magnetic beads attached with poly-T oligo were used to purifying mRNA from total RNA. First strand cDNA synthesis was performed using M-MuLV Reverse Transcriptase (RNase $\mathrm{H}-$ ), then second strand cDNA synthesis was synthesized using DNA polymerase I and RNase $\mathrm{H}$. The library fragments were purified with AMPure XP system (Beckman Coulter, Beverly, USA). After PCR amplification, the PCR products were purified (AMPure XP system) and library quality was assessed on the Agilent Bioanalyzer 2100 system. TruSeq PE Cluster Kit v3-cBot-HS (Illumina) was used to perform the index-coded samples clustering on a cBot Cluster Generation System based on the manufacturer's instructions. Then the index-coded samples were sequenced on an Illumina Novaseq platform and 150 bp paired-end reads were generated. Differential expression and normalized reads counts (FPKM, fragments per kilobase of gene/transcript model per million mapped fragments) were calculated using the DESeq2 R package (1.16.1).

Ethical conduct of research

The authors state that they have obtained appropriate institutional review board approval or have followed the principles outlined in the Declaration of Helsinki for all human or animal experimental investigations. In addition, for investigations involving human subjects, informed consent has been obtained from the participants involved.

\section{Statistical analysis}

Experiments in vitro and in vivo were performed at least 3 times. Results are expressed as the mean \pm standard deviation. Differences between two groups were assessed using Student t-test (two-tailed), oneway-ANOVA, or the Mann-Whitney U test, where appropriate. Kaplan-Meier survival analysis was used to compare esophageal cancer patient survival based on dichotomized CTD2017C7.1 expression levels retrieved from the TCGA database. Cox regression was applied to estimate prognosis, adjusting for multiple covariates, including age, gender, and stages. All statistical analyses were performed using the SAS 9.3 program (SAS Corp., NC, USA) and GraphPad Prism 7.0 (GraphPad software, Inc., USA). Statistically significant differences are presented as $* P<0.05$ and ${ }^{*} P<0.01$. In all cases, $P<0.05$ was considered statistically significant.

\section{Results}

CTD-2017C7.1 is up-regulated in human ESCC tissues and cell lines and correlates with poor prognosis

To investigate the importance of CTD-2017C7.1 in ESCC, we firstly analyzed the expression levels of CTD2017C7.1 in human ESCC tissues compared with normal tissues. The results showed that CTD-2017C7.1 expression was significantly higher in ESCC tissues than that in adjacent normal tissues (Fig. 1A). Furthermore, CTD-2017C7.1 expression levels were determined in ESCC cell lines and NE3 cells. The results revealed that CTD-2017C7.1 expression was increased in ESCC cell lines compared with normal 
cells (Fig. 1B). To further validate these results, we extracted data from GEPIA (http://gepia.cancerpku.cn/,a TCGA database-based analysis website), and analyzed the CTD-2017C7.1 expression levels in esophageal cancer (including both ESCC and EA subtypes). The results showed that CTD-2017C7.1 expression level in esophageal cancer tissues was significantly higher than that in normal tissues (Fig. 1C). We divided TCGA samples into high (above median) and low (below median) CTD-2017C7.1 expression groups based on the median value of CTD-2017C7.1 expression levels. Kaplan-Meier survival analysis was used to examine the association between CTD-2017C7.1 expression and esophageal cancer patient prognosis. Notably, patients with higher CTD-2017C7.1 expression levels had significantly shorter overall survival time than those expressing lower levels of CTD-2017C7.1 (log-rank P =0.039) (Fig. 1D). In addition, multivariate Cox regression analysis verified that higher expression of CTD-2017C7.1 was correlated with lower survival probability $(H R=1.70, P=0.04$; Fig. 1D).

CTD-2017C7.1 promotes ESCC cell proliferation, migration and invasion in vitro

To determine the biological functions of CTD-2017C7.1 in ESCC cells, lentiviruses were designed to establish CTD-2017C7.1-overexpression TE-1 cells and CRISPR/Cas9-mediated plasmids were constructed to silence CTD-2017C7.1 in TE-1 cells, respectively (Supplementary Fig. 1-2). The results of CCK-8 experiments showed that over-expression of CTD-2017C7.1 promoted TE-1 cell proliferation (Fig. 2A), while CTD-2017C7.1 depletion had the opposite effects in TE-1 cells (Fig. 3A). Moreover, upregulation of CTD-2017C7.1 increased cell migration rate (Fig. 2B). On the contrary, TE-1 cells with CTD2017C7.1 knockdown had significantly lower migration rates (Fig. 3B) compared with the control cells. Furthermore, wound healing assay showed that over-expression of CTD2017C7.1 significantly enhanced the invasion capacity of TE-1 cells (Fig. 2C), and silencing of CTD-2017C7.1 suppressed the invasion ability of TE-1 cells (Fig. 3C). We also assessed the impacts of CTD-2017C7.1 over-expression or knockdown on ESCC cell phenotypes, but no significant effects were observed (data not shown). Together, these results suggested that CTD-2017C7.1 may be involved in the pathogenesis of ESCC by up-regulating ESCC cell proliferation, Invasion and migration.

Over-expression of CTD-2017C7.1 enhances ESCC cell growth in vivo

To explore whether CTD-2017C7.1 would also affect tumorigenesis in vivo, TE-1 cells were transfected with CTD2017C7.1-overexpression cells or control cells, and were injected into nude mice, respectively. After 21 days of injection, the tumors formed in the CTD-2017C7.1 over-expression group were significantly larger than those in the control group (Fig. 5A, 5F, 5G). Additionally, the mean tumor weight in the CTD-2017C7.1-overexpression group was significantly higher than that of control mice (Fig. 5B). Nevertheless, no significant difference of body weight was observed between CTD-2017C7.1 overexpression group and the empty vector group (Fig. 5C). In addition, IHC assay showed that tumors formed from CTD-2017C7.1-overexpression cells exhibited increased positive Ki-67 cells than that from control cells, and HE staining showed similar changes (Fig. 5D, 5E). Collectively, these results indicated that over expression of CTD-2017C7.1 significantly promoted tumor growth in vivo.

CTD-2017C7.1 directly binds to PDIA3 in ESCC cells 
It has been reported that IncRNAs are involved in regulation of cancer cells phenotypes through activation of oncogenes or inactivation of tumor suppressor by binding to specific RNA-binding proteins[22]. To examine the interactions of CTD-2017C7.1 with potential RNA-binding proteins that regulate malignant phenotypes in ESCC cells, we performed RNA pull-down assay, followed by mass spectrometry analysis, in TE-1 cells. Silver staining of RNA pull down showed that CTD-2017C7.1 indeed bound with many proteins in TE-1 cells (Fig. 4A, Supplementary Table 2). Pathway analysis revealed that proteins identified from RNA pull down assay were mainly enriched in cancer-associated pathways such as biosynthesis of amino acids (hsa01230), Glycolysis/Gluconeogenesis (hsa00010), PI3K-Akt signaling pathway (hsa04151), Carbon metabolism (hsa01200), NOD-like receptor signaling pathway (hsa04621), Estrogen signaling pathway (hsa04915) (Fig. 4B). Among the binding proteins identified, all of the top 10 binding proteins (Fig. 4D), including PDIA3, TPI1, ANXA2P2, ANXA1, MAPK1, HSP90AB1, HSP90AA1, PKM, PDIA4, and $\mathrm{YWHAE}$, have been previously found to be associated with cancer development and progression, supporting the validity of our findings. We chose PDIA3 for further validation because PDIA3 was ranked as the top 1 binding protein in MS analysis with the highest score and its expression has been associated with ESCC pathological stage and prognosis[23, 24]. RIP experiment confirmed the existence of interaction between CTD-2017C7.1 and PDIA3 in TE-1 cells (Fig. 4C). These data demonstrated that CTD2017C7.1 regulated ESCC malignancy through interacting with its binding proteins.

CTD-2017C7.1 transcriptionally modified cancer-associated expression in ESCC cells

To investigate the molecular targets of CTD-2017C7.1 in ESCC cells, we first examined the distribution of CTD-2017C7.1 in ESCC cells. The results showed that CTD-2017C7.1 was mainly localized in the nucleus (Fig. 6A), suggesting that CTD-2017C7.1 may be involved in the transcriptional regulation of ESCC cells. To explore the potential target genes regulated by CTD-2017C7.1 in ESCC, transcriptome profiles in stably expressed CTD-2017C7.1 TE-1 cells were compared with that of control cells. RNA sequencing identified 93 up-regulated and 92 down-regulated (fold-change $\geqq 2, P<0.001$ ) genes modified by CTD-2017C7.1overexpression (Fig. 6B, Supplementary Table 3-4). Principal component analysis showed that gene expression profiles in CTD-2017C7.1 over-expression cells were distinct from that in control cells (Fig. 6C). Gene Ontology (GO) analysis revealed that up-regulation genes were more likely to be involved in cell cycle-related biological processes (Supplementary Fig. 5A), whereas down-regulated genes were obviously implicated in Wnt signaling processes (Supplementary Fig. 5B). KEGG pathway enrichment analysis suggested that up-regulated genes were enriched in tumor-associated pathways including cell cycle, RNA transport, DNA replication, and splicesome (Fig. 6D). While down-regulated genes were more concentrated in pathways such as Wnt, Hippo, Notch, and lysosome signaling pathways (Supplementary Fig. 5C). Among the top genes up-regulated by CTD-2017C7.1 (Fig. 6E), LAMA4, ABCG2, CFH, LUCAT1, and DIO2 have been reported to play important roles in the pathogenesis of cancers. Gene set enrichment analysis (GSEA) demonstrated that differentially expressed genes in CTD-2017C7.1 over-expression cells were significantly enriched in cancer-related gene sets RNA degradation, drug metabolism, HIF-1, PI3KAKT, Platinum drug resistance, cell cycle, Nucleotide excision repair (Fig. 6F-I, Supplementary Table 5-6). 


\section{Discussion}

Many IncRNAs have been reported to be associated with cancer development and progression, but only a few IncRNAs have been functionally characterized. Identification of ESCC-associated IncRNAs, investigation of their clinical significance and biological functions may facilitate the development of IncRNA-directed diagnostic strategy or therapy against this malignancy. In the present study, we identified a novel IncRNA CTD-2017C7.1 and demonstrated that it was significantly up-regulated in ESCC cell lines as well as in human ESCC tumor tissues. Increased CTD2017C7.1 expression was associated with poor prognosis of esophageal cancer patients. Moreover, higher CTD-2017C7.1 expression promoted ESCC cell proliferation and migration, while silencing of CTD-2017C7.1 suppressed the malignancy phenotypes of ESCC cells. The in vivo studies confirmed that overexpression of CTD-2017C7.1 enhanced tumor growth in ESCC cells. Furthermore, we showed that CTD-2017C7.1 exerted its oncogenic effects by binding to PDIA3 and activating expression of oncogenic genes. Collectively, these findings indicate that CTD2017C7.1 might play important roles in ESCC carcinogenesis.

CTD-2017C7. 1 is located on chromosome 14 (chr14:102299772-102305868). As a novel IncRNA recently identified by RNA-sequencing in plasma exosomes from patients with ESCC[19], the biological functions of CTD-2017C7.1 are unclear. Since prior studies have suggested that some low-expressed cellular IncRNAs were selectively enriched in exosomes[25,26], it raised the question whether measurement of exosomal IncRNA levels can reflect the real abundance or biological functions of exosomal IncRNAs in their parental cells. In the present study, we first proved that the expression levels of CTD-2017C7.1 were up-regulated in both tumor tissues and in ESCC cells. These observations were in consistent with that of results in exosomes[19]. We then demonstrated that CTD-2017C7.1 promoted ESCC malignancy in vitro and in vivo. Our findings supported the idea that exosomal IncRNAs such as CTD-2017C7.1 were functional representatives of cellular IncRNAs in ESCC cells or in ESCC tumor tissues.

To explore the mechanism of action of CTD-2017C7.1, we conducted RNA pull-down assays and MS analysis, which led to identification of several cancer-associated binding proteins. Among them, PDIA3 was ranked as the top 1 binding protein with the highest score and its bind to CTD-2017C7.1 was confirmed by RIP analysis. PDIA3, also known as ERp57, is a multifunctional member of the PDI family, exhibiting redox and protein disulfide isomerase activity[27]. PDIA3 is a molecular chaperone that was found to be associated with transcript factors responsible for expression regulation of genes involved in proliferation, apoptosis and oncogenesis[28]. PDIA3 was found in many different subcellular locations[29, 30]. Containing a nuclear location sequence KKKK[31], PDIA3 can translocate to nuclear from cytoplasm when the cell was stimulated by TNF-a or NF-KB[32, 33]. Dysregulation of PDIA3 was involved in a variety of disorders including cancer[34,35]. It was found that up-regulation of PDIA3 promoted cancer cell proliferation[36], while downregulation of PDIA3 suppressed cell growth[37]. Some studies revealed that PDIA3 activated AP-1 through participation in nuclear protein complexes in melanoma, and lymphoma cell lines[38]. PDIA3 was also able to interact with NF-KB[33], which promoted cell cycle progression by regulating the expression of genes involved in the cell cycle machinery such as 
CDK [39] and cyclins[40]. Consistent with previous reports that IncRNAs can regulate gene expression by interacting with RNA binding proteins[41, 42], the present study found that CTD-2017C7.1 specifically bound to PDIA3 in ESCC cells, suggesting that CTD-2017C7.1 might be involved in the pathogenesis of ESCC, at least in part, by interaction with PDIA3. Further studies are required to elucidate the detailed underlying mechanisms of CTD-2017C7.1-PDIA3 interaction in ESCC cells.

Increasing evidence has revealed that IncRNAs contribute to tumorigenesis by silencing tumor suppressor genes or activating oncogenes[15, 21]. Since CTD-2017C7.1 mainly localized in the nucleus, RNA sequencing was performed to identify target genes transcriptionally regulated by CTD-2017C7.1. Transcriptome profiling showed that CTD-2017C7.1 activated differential expression of genes involving in cancer-associated pathways, such as Wnt, Hippo, cell cycle, and DNA replication pathways. Among the top CTD-2017 C7.1-regulated genes, several of them, such as LAMA4, ABCG2, CFH, LUCAT1, and DIO2, have been reported to be involved in cancer development and progression. LAMA4 is a gene functionally associated with PI3K-AKT signaling pathway. Up-regulation of LAMA4 has been correlated with high metastasis potential and poor survival outcome of pancreatic cancer[43]. Abnormally high expression of ABCG2 in esophageal cancer tissues is involved in multidrug resistance of esophageal cancer [44] and gastric cancer[45]. CFH participated in the maintenance of stemness in liver tumor-initiating cells[46]. DIO2 activation was also associated with poor clinical outcomes and treatment resistance[47]. Higher expression level of LUCAT1 can predict a shorter survival time of patients in different cancers[48]. Importantly, LUCAT1 could regulate the stability of DNMT1 and inhibit the expression of tumor suppressors through DNA methylation, leading to the formation and metastasis of ESCC, indicating that LUCAT1 may serve as a potential therapeutic target and as a biomarker for ESCC[49]. Taken together, these findings suggested that CTD-2017C7.1 might act in concert with LAMA4, ABCG2, CFH, LUCAT1, $\mathrm{DIO} 2$, and other oncogenic genes to promote tumorigenesis of ESCC cells. But future studies are needed to clarify CTD-2017C7.1 regulation with other potential target genes and the detailed molecular mechanisms underlying regulatory behaviors in ESCC cells.

\section{Conclusion}

The present study provided evidence that the ESCC-associated novel exosomal IncRNA CTD-2017C7.1 is an oncogenic IncRNA that promotes tumorigenesis of ESCC through binding PDIA3 and activating oncogenic gene expressions. These findings improve our understanding of ESCC pathogenesis, and facilitate the development of IncRNA-directed diagnostics and therapeutics against ESCC.

\section{Abbreviations}

IncRNAs

Long noncoding RNAs;ESCC:esophageal squamous cell carcinoma; MS:Mass Spectrometry; RIP:RNA Binding Protein Immunoprecipitatio; qRT-PCR:Real-Time Quantitative PCR; CRISPR:Clustered Regularly Interspaced Short Palindromic Repeats; PDIA3:protein disulfide isomerase family A member 3; EA:esophageal adenocarcinoma; ZFAS1:Zinc finger antisense 1; PCAT-1:prostate cancer-associated 
transcript 1; MALAT1:metastasis-associated lung adenocarcinoma transcript 1; DDP:cisplatin; ATCC:american type culture collection; HE:hematoxylin-eosin; IHC:immunohistochemistry; TPI1:triosephosphate isomerase 1; ANXA2P2:annexin A2 pseudogene 2; ANXA1:annexin a1; MAPK1:mitogen-activated protein kinase 1; HSP90AB1:heat-shock protein, 90-kd, alpha, class b, member 1; HSP90AA1:heat-shock protein, 90-kd, alpha, class a, member 1; PKM:pyruvate kinase, muscle; PDIA4:protein disulfide isomerase family A member 4; TNF-a:tumor necrosis factor-a; NF-kB:necrosis factor-KB; CDK:cyclin-dependent kinase; LAMA4:laminin, alpha-4; ABCG2:atp-binding cassette, subfamily g, member 2; CFH:complement factor h; LUCAT1:lung cancer-associated transcript 1; DIO2:deiodinase, iodothyronine, type 2 .

\section{Declarations}

\section{Ethics approval and consent to participate}

The study was approved by the Ethics Review Committee of Shenzhen University Health Science Center. The animal study followed the Guidelines for the Animal Care and Use approved by Shenzhen University Health Science Center. (1. Ethics approval and consent to participate)

\section{Consent for publication}

Not applicable. (2. Consent for publication)

\section{Availability of data and materials}

The datasets used and/or analyzed during the current study are available from the corresponding author on reasonable request. (3. Availability of data and materials)

\section{Competing interests}

Authors declare no conflicts of interest for this article. (4. Competing interests)

\section{Funding}

This study was supported by the National Natural Science Foundation of China (NSFC) (81572908), and the Peacock Initiative Program of Shenzhen Municipality (827-000157), Top University Construction Project (SZU. 86000000210). Shenzhen Science and Innovation Commission (KQCX2015032416521307). (5. Funding)

\section{Authors' contributions}

Rihong Zhai designed the study; Peikun Ding, Kang Zhang, Wenhan Yang, Qianqian Chen, Yi Song, Guogang Cheng, Lin Yang, Shaowu Ye, Liyuan Sun and Liang Wang performed the experiments and prepared the figures; Rihong Zhai and Liang Wang contributed to drafting the manuscript. All authors read and approved the final manuscript. (6. Authors' contributions) 
Acknowledgements

Not applicable. (7. Acknowledgements)

\section{References}

1. Bray F, Ren JS, Masuyer E, Ferlay J. Global estimates of cancer prevalence for 27 sites in the adult population in 2008. Int J Cancer. 2013;132(5):1133-45.

2. Abnet CC, Arnold M, Wei WQ. Epidemiology of Esophageal Squamous Cell Carcinoma. Gastroenterology. 2018;154(2):360-73.

3. Smyth EC, Lagergren J, Fitzgerald RC, Lordick F, Shah MA, Lagergren P, Cunningham D. Oesophageal cancer. Nat Rev Dis Primers. 2017;3:17048.

4. Wu C, Wang Z, Song X, Feng XS, Abnet CC, He J, Hu N, Zuo XB, Tan W, Zhan Q, et al. Joint analysis of three genome-wide association studies of esophageal squamous cell carcinoma in Chinese populations. Nat Genet. 2014;46(9):1001-6.

5. Businello G, Parente P, Mastracci L, Pennelli G, Traverso G, Milione M, Bellan E, Michelotto M, Kotsafti A, Grillo F, et al: The Pathologic and Molecular Landscape of Esophageal Squamous Cell Carcinogenesis. Cancers (Basel) 2020, 12(8).

6. Batista PJ, Chang HY. Long noncoding RNAs: cellular address codes in development and disease. Cell. 2013;152(6):1298-307.

7. Cech TR, Steitz JA. The noncoding RNA revolution-trashing old rules to forge new ones. Cell. 2014;157(1):77-94.

8. Ulitsky I, Bartel DP. lincRNAs: genomics, evolution, and mechanisms. Cell. 2013;154(1):26-46.

9. Xu Y, Zhang X, Hu X, Zhou W, Zhang P, Zhang J, Yang S, Liu Y. The effects of IncRNA MALAT1 on proliferation, invasion and migration in colorectal cancer through regulating SOX9. Mol Med. 2018;24(1):52.

10. Liu M, Zhang H, Li Y, Wang R, Li Y, Zhang H, Ren D, Liu H, Kang C, Chen J. HOTAIR, a long noncoding RNA, is a marker of abnormal cell cycle regulation in lung cancer. Cancer Sci. 2018;109(9):2717-33.

11. Huarte M. The emerging role of IncRNAs in cancer. Nat Med. 2015;21(11):1253-61.

12. Iyer MK, Niknafs YS, Malik R, Singhal U, Sahu A, Hosono Y, Barrette TR, Prensner JR, Evans JR, Zhao $S$, et al. The landscape of long noncoding RNAs in the human transcriptome. Nat Genet. 2015;47(3):199-208.

13. Shi H, Liu Z, Pei D, Jiang Y, Zhu H, Chen B. Development and validation of nomogram based on IncRNA ZFAS1 for predicting survival in lymph node-negative esophageal squamous cell carcinoma patients. Oncotarget. 2017;8(35):59048-57.

14. Zhang F, Wan M, Xu Y, Li Z, Leng K, Kang P, Cui Y, Jiang X. Long noncoding RNA PCAT1 regulates extrahepatic cholangiocarcinoma progression via the Wnt/beta-catenin-signaling pathway. Biomed Pharmacother. 2017;94:55-62. 
15. Hu L, Wu Y, Tan D, Meng H, Wang K, Bai Y, Yang K. Up-regulation of long noncoding RNA MALAT1 contributes to proliferation and metastasis in esophageal squamous cell carcinoma. J Exp Clin Cancer Res. 2015;34:7.

16. Liu J, Mayekar MK, Wu W, Yan M, Guan H, Wang J, Zaman A, Cui Y, Bivona TG, Choudhry H, et al. Long non-coding RNA ESCCAL-1 promotes esophageal squamous cell carcinoma by down regulating the negative regulator of APOBEC3G. Cancer Lett. 2020;493:217-27.

17. Wang G, Feng B, Niu Y, Wu J, Yang Y, Shen S, Guo Y, Liang J, Guo W, Dong Z. A novel long noncoding RNA, LOC440173, promotes the progression of esophageal squamous cell carcinoma by modulating the miR-30d-5p/HDAC9 axis and the epithelial-mesenchymal transition. Mol Carcinog. 2020;59(12):1392-408.

18. Liu G, Guo W, Chen G, Li W, Cui Y, Qin J, Peng J. Lnc-MCEI mediated the chemosensitivity of esophageal squamous cell carcinoma via miR-6759-5p to competitively regulate IGF2. Int J Biol Sci. 2020;16(15):2938-50.

19. Tian L, Yang L, Zheng W, Hu Y, Ding P, Wang Z, Zheng D, Fu L, Chen B, Xiao T, et al. RNA sequencing of exosomes revealed differentially expressed long noncoding RNAs in early-stage esophageal squamous cell carcinoma and benign esophagitis. Epigenomics. 2020;12(6):525-41.

20. Xiong JX, Wang YS, Sheng J, Xiang D, Huang TX, Tan BB, Zeng CM, Li HH, Yang J, Meltzer SJ, et al. Epigenetic alterations of a novel antioxidant gene SLC22A3 predispose susceptible individuals to increased risk of esophageal cancer. Int J Biol Sci. 2018;14(12):1658-68.

21. Yang W, Qian Y, Gao K, Zheng W, Wu G, He Q, Chen Q, Song Y, Wang L, Wang Y, et al: LncRNA BRCAT54 inhibits the tumorigenesis of non-small cell lung cancer by binding to RPS9 to
transcriptionally regulate JAK-STAT and calcium pathway genes. Carcinogenesis 2020 .

22. Bartonicek N, Maag JL, Dinger ME. Long noncoding RNAs in cancer: mechanisms of action and technological advancements. Mol Cancer. 2016;15(1):43.

23. Zeng FM, He JZ, Wang SH, Liu DK, Xu XE, Wu JY, Li EM, Xu LY. A Novel Three-Gene Model Predicts Prognosis and Therapeutic Sensitivity in Esophageal Squamous Cell Carcinoma. Biomed Res Int. 2019;2019:9828637.

24. He JZ, Wu ZY, Wang SH, Ji X, Yang CX, Xu XE, Liao LD, Wu JY, Li EM, Zhang K, et al. A decision treebased combination of ezrin-interacting proteins to estimate the prognostic risk of patients with esophageal squamous cell carcinoma. Hum Pathol. 2017;66:115-25.

25. Kogure T, Yan IK, Lin WL, Patel T. Extracellular Vesicle-Mediated Transfer of a Novel Long Noncoding RNA TUC339: A Mechanism of Intercellular Signaling in Human Hepatocellular Cancer. Genes Cancer. 2013;4(7-8):261-72.

26. Gezer U, Ozgur E, Cetinkaya M, Isin M, Dalay N. Long non-coding RNAs with low expression levels in cells are enriched in secreted exosomes. Cell Biol Int. 2014;38(9):1076-9.

27. Takata H, Kudo M, Yamamoto T, Ueda J, Ishino K, Peng WX, Wada R, Taniai N, Yoshida H, Uchida E, et al. Increased expression of PDIA3 and its association with cancer cell proliferation and poor prognosis in hepatocellular carcinoma. Oncol Lett. 2016;12(6):4896-904. 
28. Hettinghouse A, Liu R, Liu CJ. Multifunctional molecule ERp57: From cancer to neurodegenerative diseases. Pharmacol Ther. 2018;181:34-48.

29. Hatahet F, Ruddock LW. Protein disulfide isomerase: a critical evaluation of its function in disulfide bond formation. Antioxid Redox Signal. 2009;11(11):2807-50.

30. Raykhel I, Alanen H, Salo K, Jurvansuu J, Nguyen VD, Latva-Ranta M, Ruddock L. A molecular specificity code for the three mammalian KDEL receptors. J Cell Biol. 2007;179(6):1193-204.

31. Adikesavan AK, Unni E, Jaiswal AK. RETRACTED: Overlapping signal sequences control nuclear localization and endoplasmic reticulum retention of GRP58. Biochem Biophys Res Commun. 2008;377(2):407-12.

32. Grindel BJ, Rohe B, Safford SE, Bennett JJ, Farach-Carson MC. Tumor necrosis factor-alpha treatment of HepG2 cells mobilizes a cytoplasmic pool of ERp57/1,25D(3)-MARRS to the nucleus. J Cell Biochem. 2011;112(9):2606-15.

33. Wu W, Beilhartz G, Roy Y, Richard CL, Curtin M, Brown L, Cadieux D, Coppolino M, Farach-Carson MC, Nemere I, et al. Nuclear translocation of the 1,25D3-MARRS (membrane associated rapid response to steroids) receptor protein and NFkappaB in differentiating NB4 leukemia cells. Exp Cell Res. 2010;316(7):1101-8.

34. Choe MH, Min JW, Jeon HB, Cho DH, Oh JS, Lee HG, Hwang SG, An S, Han YH, Kim JS. ERp57 modulates STAT3 activity in radioresistant laryngeal cancer cells and serves as a prognostic marker for laryngeal cancer. Oncotarget. 2015;6(5):2654-66.

35. Wise R, Duhachek-Muggy S, Qi Y, Zolkiewski M, Zolkiewska A. Protein disulfide isomerases in the endoplasmic reticulum promote anchorage-independent growth of breast cancer cells. Breast Cancer Res Treat. 2016;157(2):241-52.

36. Liu Y, Wang JX, Nie ZY, Wen Y, Jia XJ, Zhang LN, Duan HJ, Shi YH. Upregulation of ERp57 promotes clear cell renal cell carcinoma progression by initiating a STAT3/ILF3 feedback loop. J Exp Clin Cancer Res. 2019;38(1):439.

37. Kondo R, Ishino K, Wada R, Takata H, Peng WX, Kudo M, Kure S, Kaneya Y, Taniai N, Yoshida H, et al. Downregulation of protein disulfideisomerase A3 expression inhibits cell proliferation and induces apoptosis through STAT3 signaling in hepatocellular carcinoma. Int J Oncol. 2019;54(4):1409-21.

38. Aureli C, Gaucci E, Arcangeli V, Grillo C, Eufemi M, Chichiarelli S. ERp57/PDIA3 binds specific DNA fragments in a melanoma cell line. Gene. 2013;524(2):390-5.

39. Perkins ND, Felzien LK, Betts JC, Leung K, Beach DH, Nabel GJ. Regulation of NF-kappaB by cyclindependent kinases associated with the p300 coactivator. Science. 1997;275(5299):523-7.

40. Hinz M, Loser P, Mathas S, Krappmann D, Dorken B, Scheidereit C. Constitutive NF-kappaB maintains high expression of a characteristic gene network, including CD40, CD86, and a set of antiapoptotic genes in Hodgkin/Reed-Sternberg cells. Blood. 2001;97(9):2798-807.

41. Krchnakova Z, Thakur PK, Krausova M, Bieberstein N, Haberman N, Muller-McNicoll M, Stanek D. Splicing of long non-coding RNAs primarily depends on polypyrimidine tract and 5 ' splice-site sequences due to weak interactions with SR proteins. Nucleic Acids Res. 2019;47(2):911-28. 
42. Lin Z, Hu Y, Lai S, Xue M, Lin J, Qian Y, Zhuo W, Chen S, Si J, Wang L. Long Noncoding RNA: its partners and their roles in cancer. Neoplasma. 2015;62(6):846-54.

43. Zheng B, Qu J, Ohuchida K, Feng H, Chong SJF, Yan Z, Piao Y, Liu P, Sheng N, Eguchi D, et al. LAMA4 upregulation is associated with high liver metastasis potential and poor survival outcome of Pancreatic Cancer. Theranostics. 2020;10(22):10274-89.

44. Wang L, Liu L, Chen Y, Du Y, Wang J, Liu J. Correlation between adenosine triphosphate (ATP)binding cassette transporter $\mathrm{G} 2$ (ABCG2) and drug resistance of esophageal cancer and reversal of drug resistance by artesunate. Pathol Res Pract. 2018;214(9):1467-73.

45. Yu B, Gu D, Zhang X, Liu B, Xie J. The role of GLI2-ABCG2 signaling axis for 5 Fu resistance in gastric cancer. J Genet Genomics. 2017;44(8):375-83.

46. Seol HS, Lee SE, Song JS, Rhee JK, Singh SR, Chang S, Jang SJ. Complement proteins C7 and CFH control the stemness of liver cancer cells via LSF-1. Cancer Lett. 2016;372(1):24-35.

47. Hepburn AC, Steele RE, Veeratterapillay R, Wilson L, Kounatidou EE, Barnard A, Berry P, Cassidy JR, Moad M, El-Sherif A, et al. The induction of core pluripotency master regulators in cancers defines poor clinical outcomes and treatment resistance. Oncogene. 2019;38(22):4412-24.

48. Lv P, Ye T, Yang X, Liu H, Ye Z. High expression of long noncoding RNA LUCAT1 correlates with a poor clinical outcome in solid tumors: A systematic review and meta-analysis. Pathol Res Pract. 2020;216(9):153047.

49. Yoon JH, You BH, Park CH, Kim YJ, Nam JW, Lee SK. The long noncoding RNA LUCAT1 promotes tumorigenesis by controlling ubiquitination and stability of DNA methyltransferase 1 in esophageal squamous cell carcinoma. Cancer Lett. 2018;417:47-57.

\section{Figures}



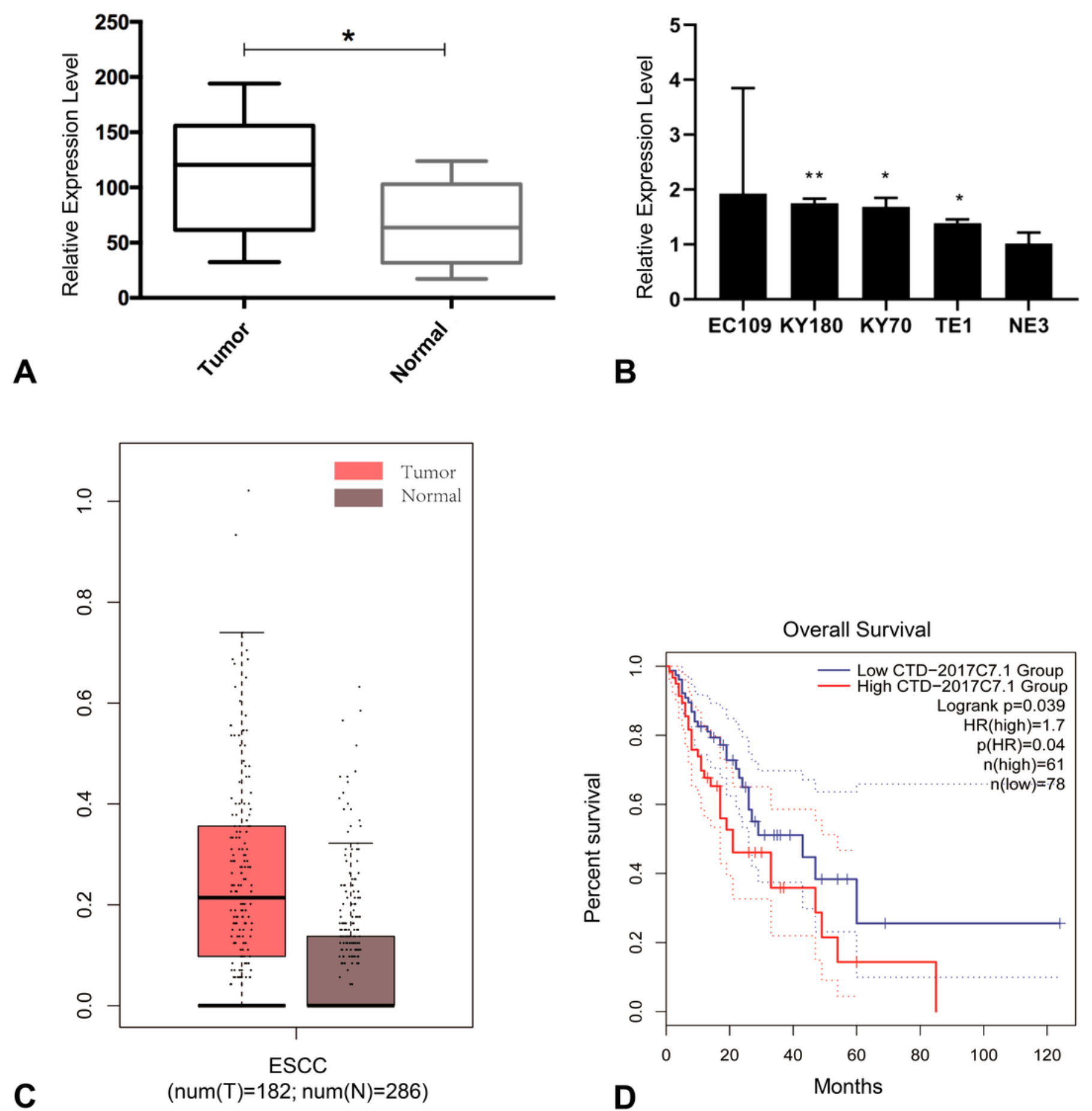

Figure 1

CTD-2017C7.1 was up-regulated in ESCC tissues and ESCC cell lines and higher expression of CTD2017C7.1 was associated with poor prognosis in esophageal cancer patients. (A) CTD-2017C7.1 expression level in ESCC tumors was significantly higher than that in adjacent normal tissues $(n=9)$. (B) CTD-2017C7.1 was up-regulated in ESCC cell lines compared with that in normal esophageal epithelial cells. (C) Higher expression of CTD-2017C7.1 was detected in esophageal cancer tissues $(n=182)$ 
compared with normal tissues $(n=286)$. The TCGA dataset was used for the analysis. (D) Kaplan-Meier survival curves revealed an association of higher CTD-2017C7.1 expression with shorter overall survival in esophageal cancer patients (log rank $P=0.039$ ). CTD-2017C7.1 expression data were downloaded from TCGA databases. All experiments were performed in at least biological triplicates and data presented as mean $\pm S D$. ${ }^{*} P<0.05,{ }^{*} P<0.01$.

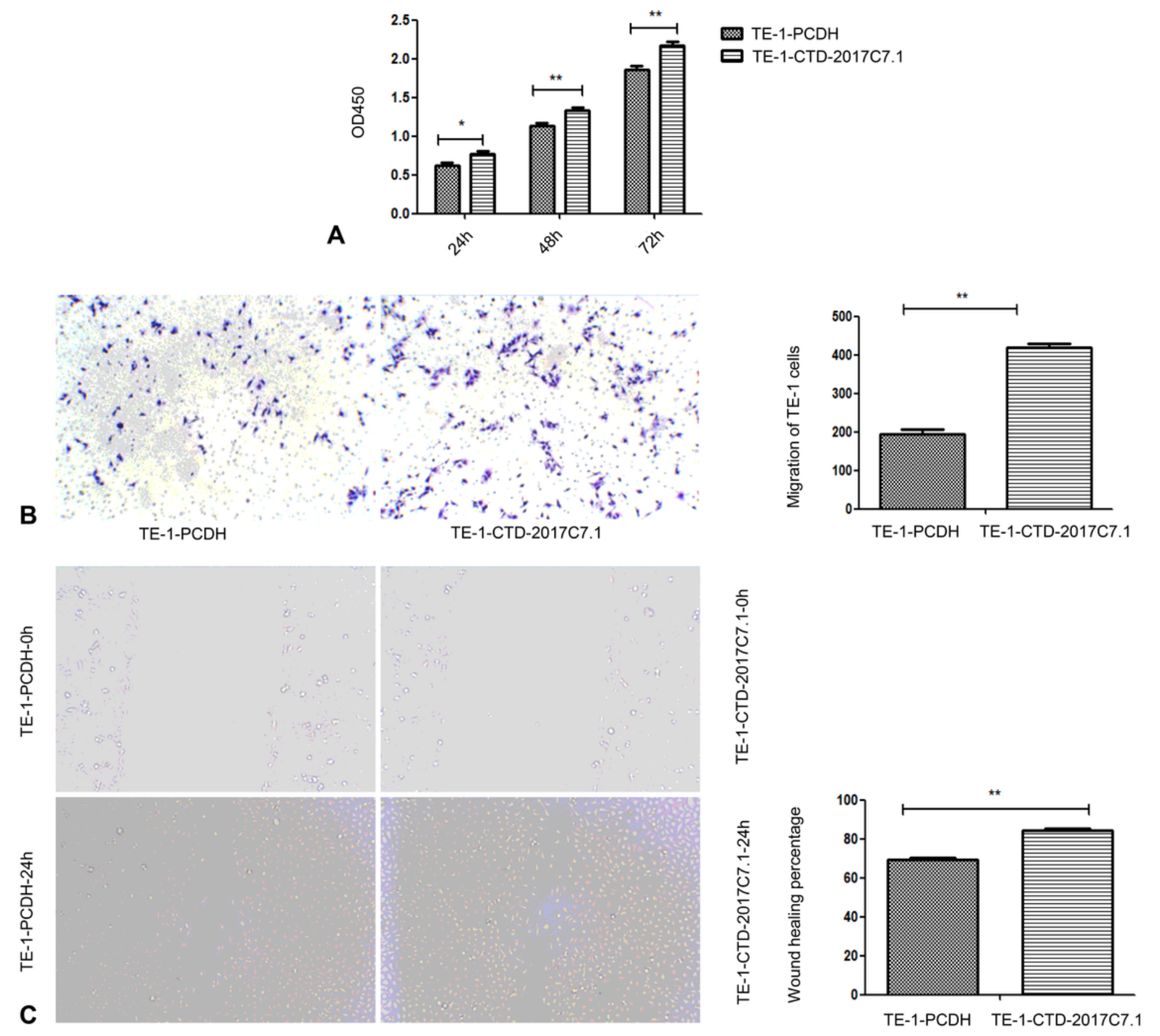

Figure 2

Over-expression of CTD-2017C7.1 promoted cell proliferation, invasion and migration of ESCC cells. (A) CCK-8 assays were used to determine the cell viability for TE-1 cells stably expressed CTD-2017C7.1 or TE-1-empty vector control cells. (B) Transwell assays were conducted to evaluate the effect of upregulation of CTD-2017C7.1 on cell invasion; cell lines were treated the same as in (A). (C) Cell migration 
was monitored using wound healing assays; cell lines were treated the same as in (A). All experiments were performed in at least biological triplicates and data presented as mean $\pm S D$. ${ }^{*} P<0.05,{ }^{* *} P<0.01$.

A

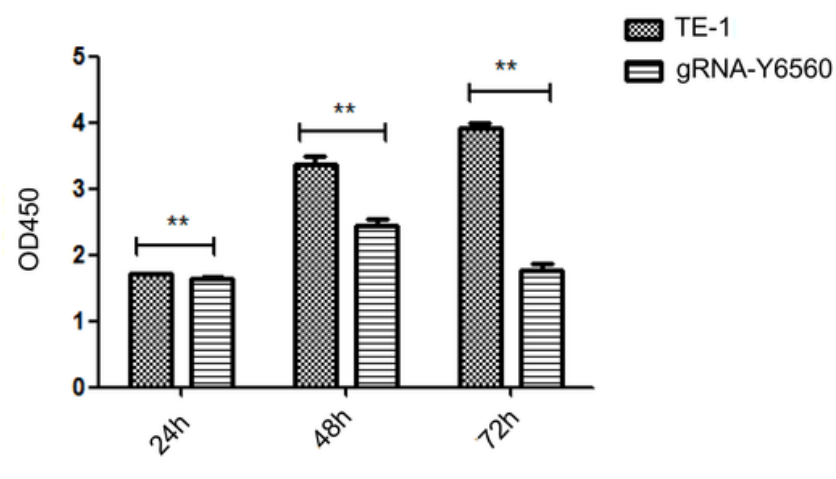

B
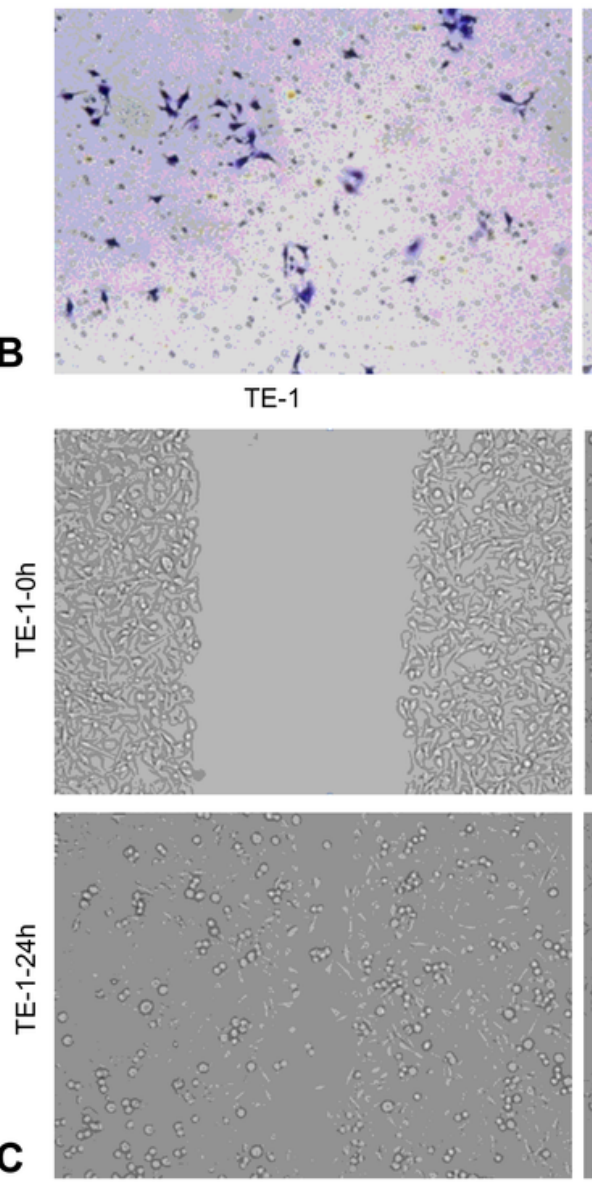
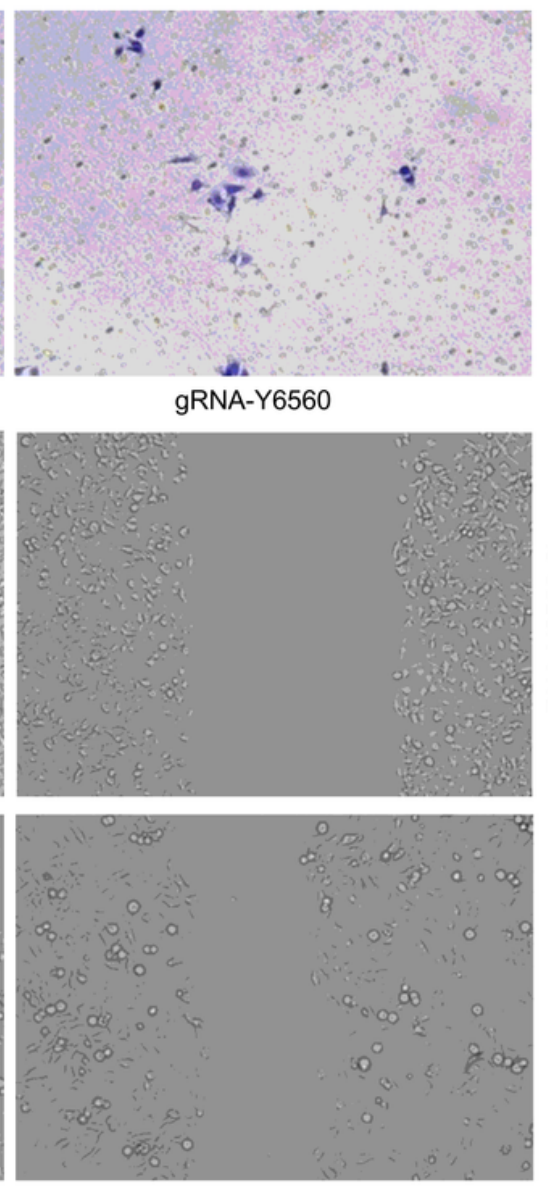
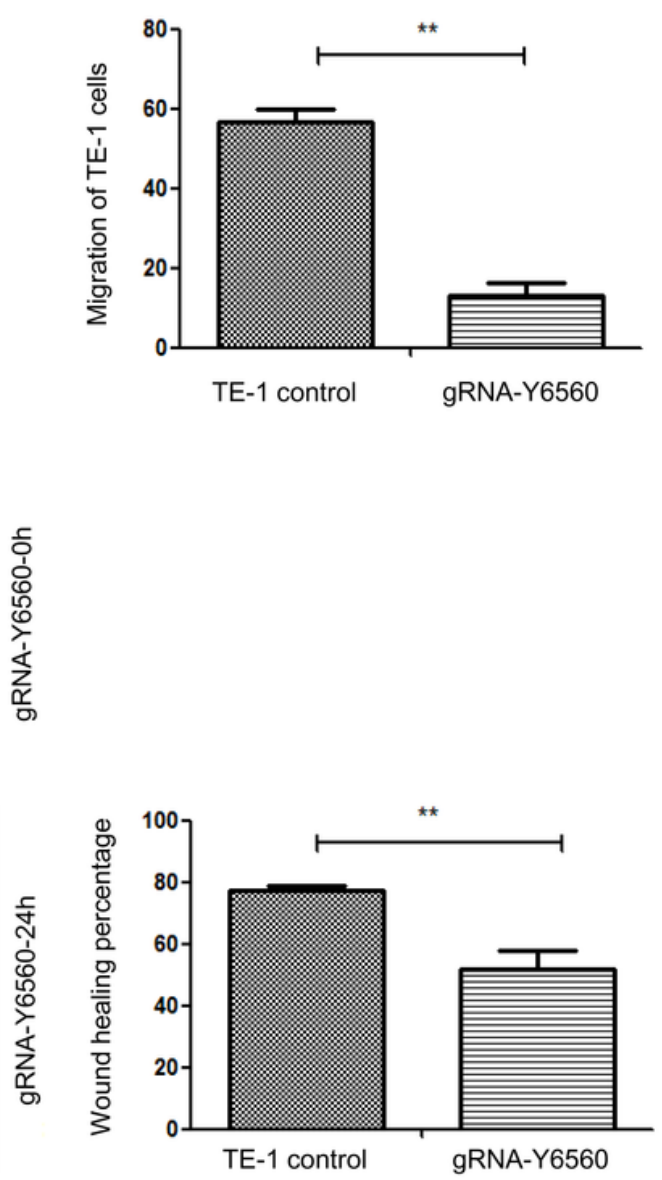

\section{Figure 3}

Knockdown of CTD-2017C7.1 inhibited cell proliferation, invasion and migration of ESCC cell. The effects of CTD-2017C7.1 depletion on the proliferation rate of were examined in TE-1 cells transfected with gRNA or NC controls. (A) Depletion of CTD-2017C7.1 significantly suppressed cell proliferation compared to that of control cells. (B) Knockdown of CTD-2017C7.1 inhibited invasion capacity of ESCC cells. (C) Wound-healing assays showed that silencing of CTD-2017C7.1 reduced migration ability of ESCC cells. All experiments were performed in at least biological triplicates and data presented as mean $\pm S D .{ }^{*} P<0.05$, $\star \star P<0.01$. 
A

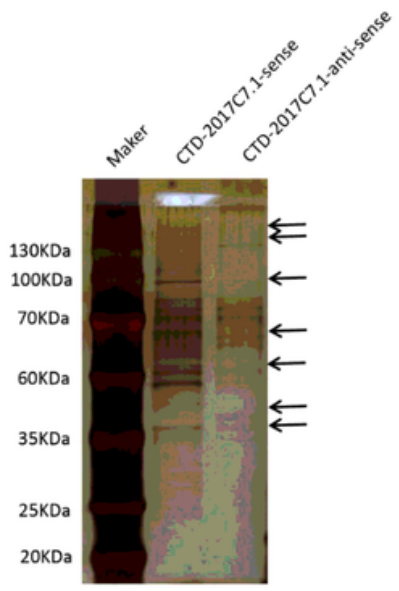

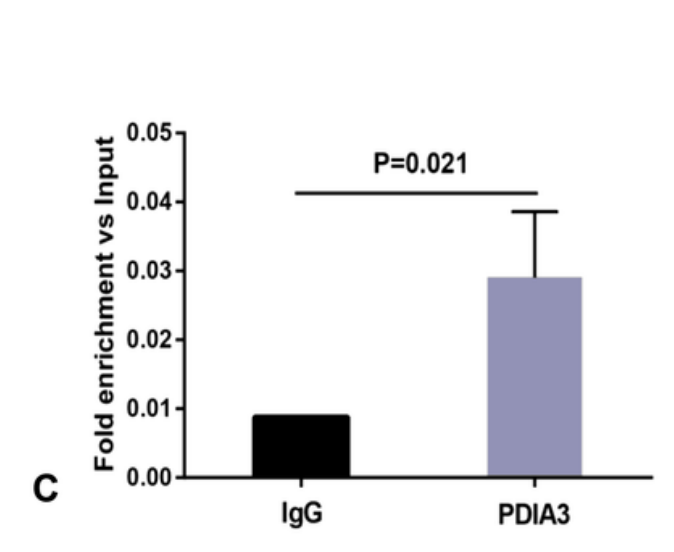

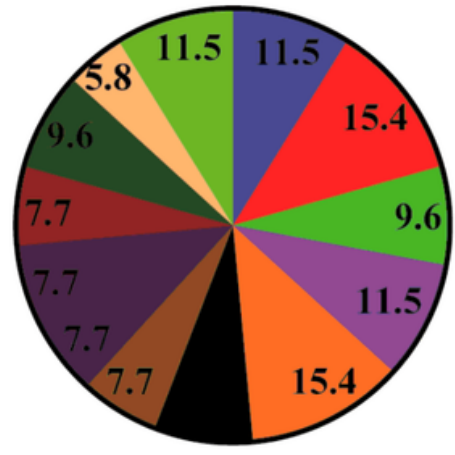

B
- hsa01230: Biosynthesis of amino acids

hsa01130: Biosynthesis of antibiotics

- hsa00010: Glycolysis / Gluconeogenesis

hsa04141: Protein processing in endoplasmic reticulum

hsa04151: PI3K-Akt signaling pathway

- hsa01200: Carbon metabolism

hsa04621: NOD-like receptor signaling pathway

nsa05215: Prostate cancer

- hsa04915: Estrogen signaling pathway

- hsa04114: Oocyte meiosis

- hsa05203: Viral carcinogenesis

hsa05230: Central carbon metabolism in cancer

hsa05200: Pathways in cancer

\begin{tabular}{|c|c|c|c|c|c|}
\hline Accession & Protein Name & Score & $\begin{array}{l}\text { \# Unique } \\
\text { Peptides }\end{array}$ & \# PSMs & MW [kDa] \\
\hline P30101 & PDIA3 & 117.55 & 4 & 4 & 56.75 \\
\hline P60174 & TPI1 & 104.53 & 1 & 1 & 30.77 \\
\hline A6NMY6 & ANXA2P2 & 100.52 & 2 & 2 & 38.63 \\
\hline P04083 & ANXA1 & 99.70 & 2 & 2 & 38.69 \\
\hline P28482 & MAPK1 & 97.20 & 1 & 2 & 41.36 \\
\hline P08238 & HSP90AB1 & 74.92 & 2 & 5 & 83.21 \\
\hline P07900 & HSP90AA1 & 74.23 & 3 & 6 & 84.61 \\
\hline P14618 & PKM & 68.06 & 2 & 2 & 57.90 \\
\hline P13667 & PDIA4 & 67.73 & 2 & 2 & 72.89 \\
\hline P62258 & YWHAE & 63.27 & 1 & 2 & 29.16 \\
\hline
\end{tabular}

\section{Figure 4}

CTD-2017C7.1 bound to PDIA3. (A) SDS-PAGE image of proteins immunoprecipitated by CTD-2017C7.1 and its antisense RNA in TE-1 cells. Arrows indicated the bands that were different between sense and antisense. (B) Pathway analysis of the CTD-2017C7.1-binding proteins. (C) RIP analysis followed by qRTPCR assay showed that CTD-2017C7.1 was specifically bound to PDIA3 protein in TE-1 cells. (D) CTD2017C7.1-binding proteins with top matching scores identified by mass spectrometry. 

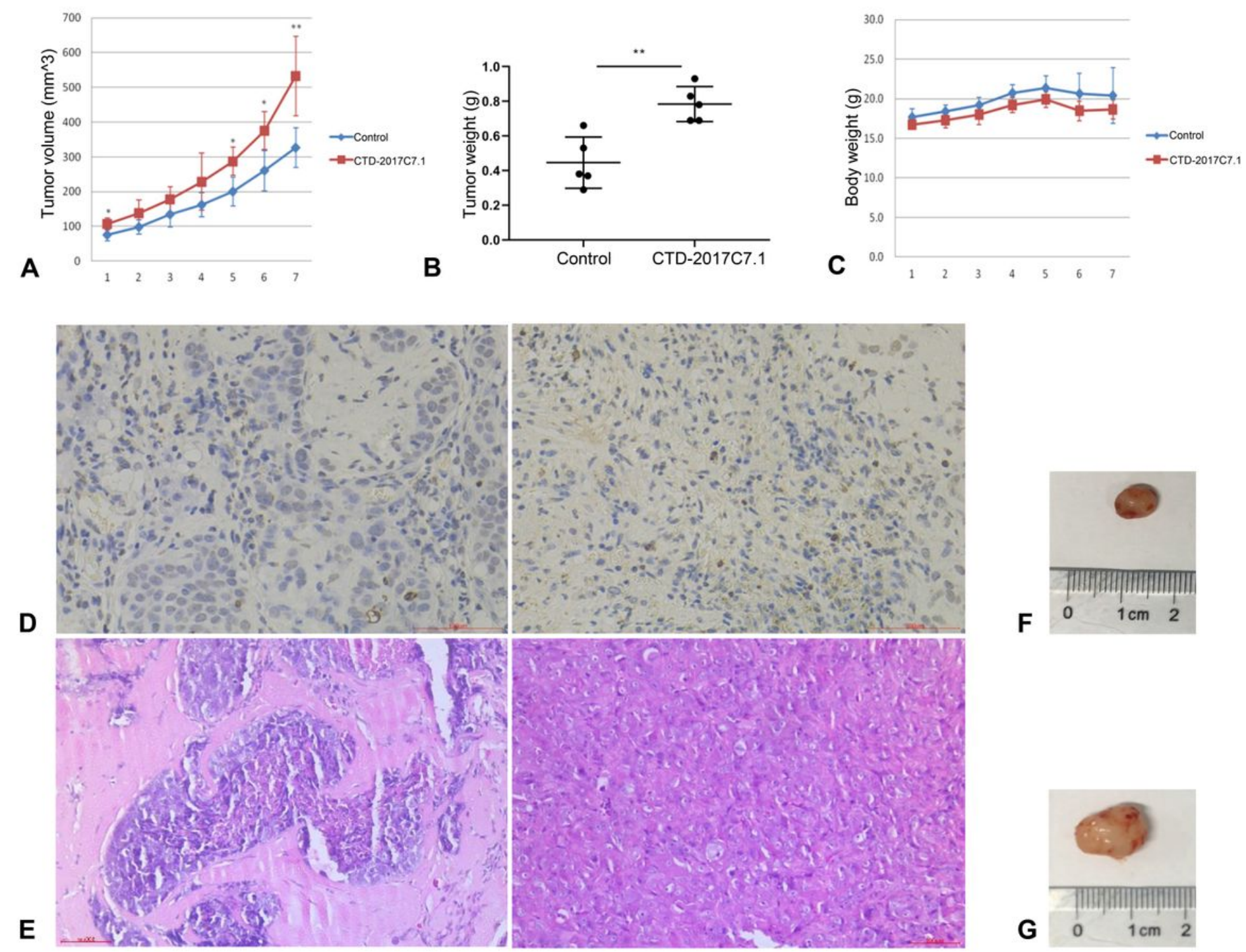

\section{Figure 5}

CTD-2017C7.1 promoted tumor growth in vivo. TE-1 cells stably expressed CTD-2017C7.1 or NC cells were injected into nude mice, respectively. (A) CTD-2017C7.1 over-expression led to higher tumor volumes than that of control mice (tumor volumes were calculated every 3 days after injection). (B) Higher expression of CTD-2017C7.1 increased tumor weight, presenting as the means of tumor weights \pm SD. (C) No significant difference of body weight was observed between CTD-2017C7.1 over-expression group and the empty vector group. (D) IHC staining showed a typical ESCC pattern in both the CTD-2017C7.1 overexpression and NC-control groups. (E) HE staining showed the increase in $\mathrm{Ki}-67$ expression in the CTD-2017C7.1-overexpression group compared to that in the NC--control group. (F) Representative image of ESCC xenograft in nude mice with NC control ESCC cells. (G) Representative image of ESCC xenograft with CTD-2017C7.1 overexpression ESCC cells. 
A

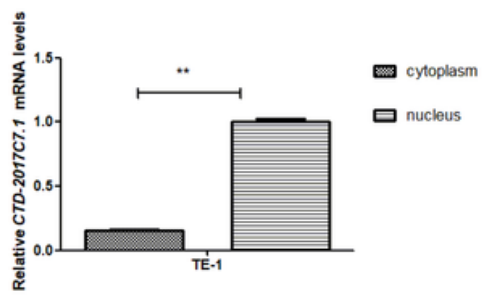

B

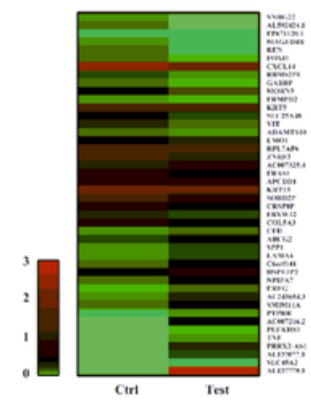

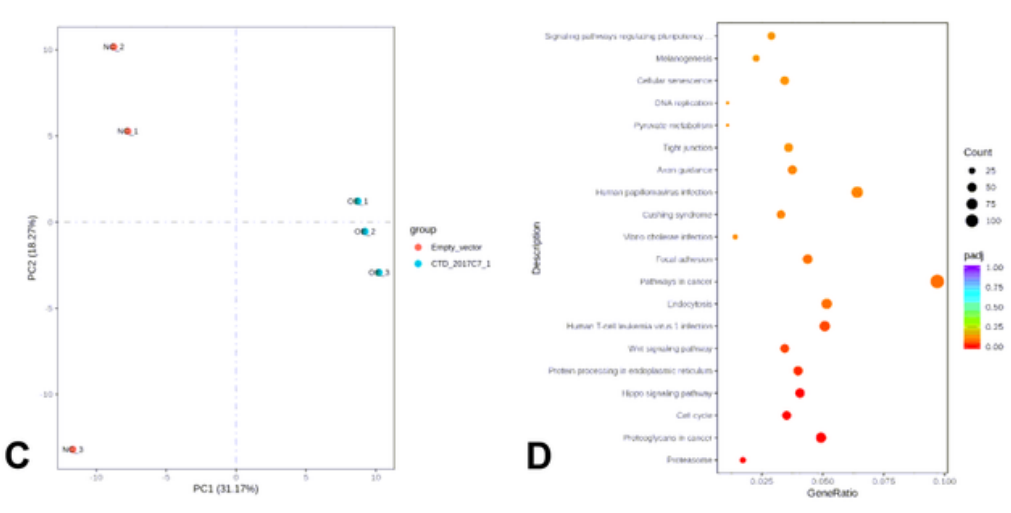
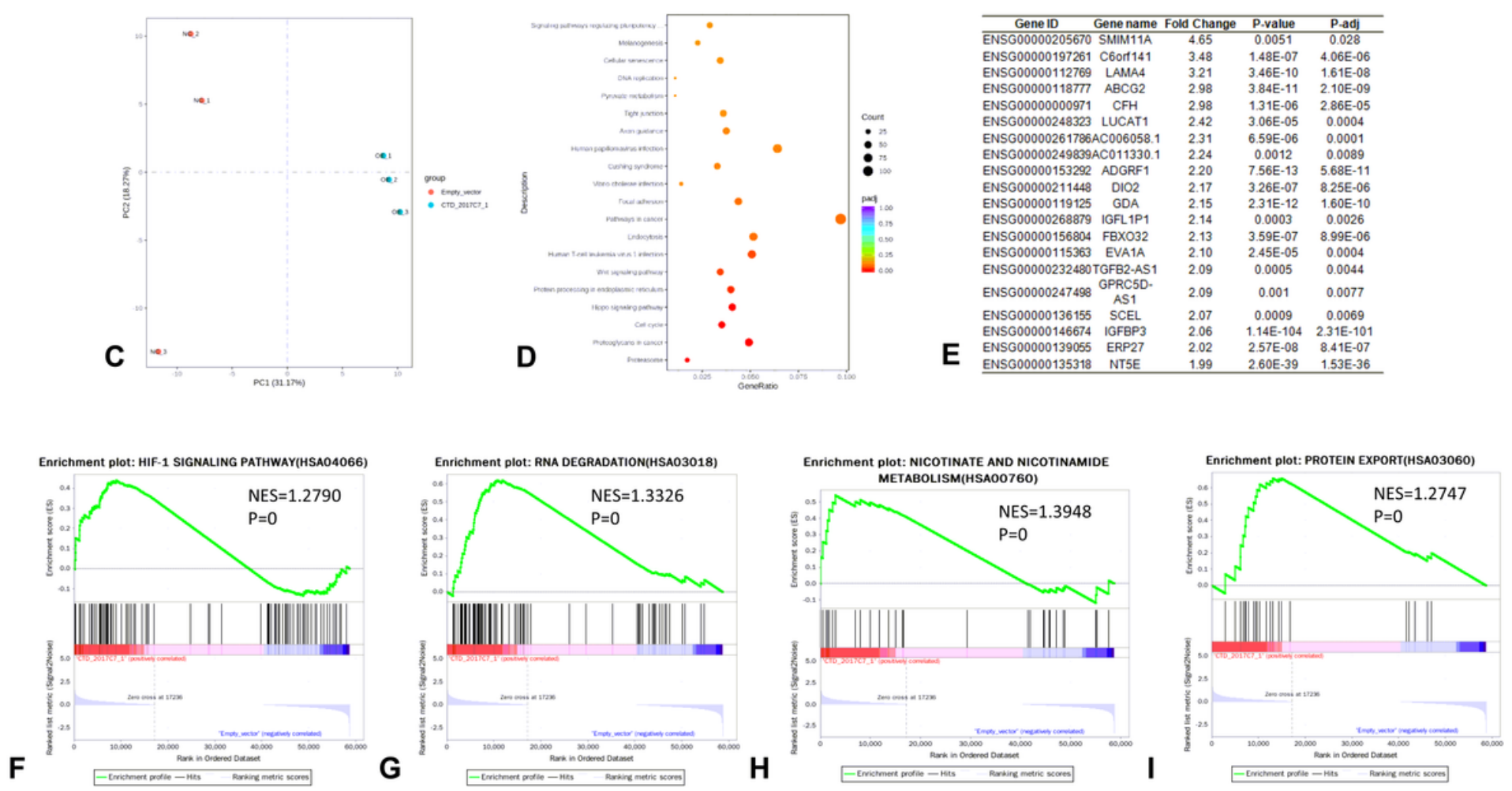

Figure 6

CTD-2017C7.1 activated oncogenic gene expressions. (A) RNA was extracted from the nuclear and the cytoplasmic fractions of TE-1 cells, and CTD-2017C7.1 was measured by qRT-PCR. The results showed that CTD-2017C7.1 was mainly localized in the nucleus. (B) Heatmap of differentially expressed genes regulated by CTD-2017C7.1 over-expression. CTD-2017C7.1 regulated genes were identified by RNA-seq. (C) Principle component analysis (PCA) analysis revealed different components of key genes between cells with CTD-2017C7.1 up-regulation and control cells. (D) KEGG analysis showed that different expression genes regulated by CTD-2017C7.1 were mainly enriched in cancer-related pathways. (E) Top 10 up-regulated genes induced by CTD-2017C7.1 over-expression. (F-I) Gene Set Enrichment Analysis (GSEA) indicated significant correlations between high expression of CTD-2017C7.1 and its up-regulated target genes.

\section{Supplementary Files}

This is a list of supplementary files associated with this preprint. Click to download. 
- SupplementaryFig1.tif

- SupplementaryFig2.tif

- SupplementaryFig3.tif

- SupplementaryFig4.tif

- SupplementaryFig5.tif 